\title{
ESTUDO COMPARATIVO DA FORÇA MUSCULAR, EQUILÍBRIO E QUALIDADE DE VIDA ENTRE IDOSOS PRATICANTES DE HIDROTERAPIA E IDOSOS SEDENTÁRIOS DO MUNICÍPIO DE CUIABÁ (MT)
}

\author{
COMPARATIVE STUDY OF MUSCLE STRENGTH, BALANCE AND QUALITY OF LIFE AMONG \\ ELDERLY PRACTITIONERS AND OLDER HYDROTHERAPY SEDENTARY OF CUIABÁ (MT), BRAZIL

\section{Viviane Aparecida Martins Salicio ${ }^{\mathrm{a}^{*}}$, Tainara da Silva Mattos ${ }^{\mathrm{b}^{* *}}$, Vanessa Lauriany de Arruda Brandalise $^{\mathrm{c}^{*}}$, Walkiria Shimoya-Bittencourt ${ }^{\mathrm{d}^{*}}$, Marcos Adriano Salicio ${ }^{\mathrm{e}^{*}}$}

\author{
avivimana@hotmail.com, btainara.mattos@hotmail.com, cbrandalise.v@gmail.com, dwshimoya@yahoo.com.br, emasalicio@ig.com.br \\ *Centro Universitário de Várzea Grande - Várzea Grande (MT), Brasil \\ **Universidade de Cuiabá - Cuiabá (MT), Brasil
}

Data de recebimento do artigo: 25/10/2014

Data de aceite do artigo: 07/04/2015

\section{RESUMO}

Introduçáo: $\mathrm{O}$ Brasil apresenta uma população de 15 milhóes de habitantes com idade igual ou superior a 60 anos, que tem apresentado crescimento de 5\% nos últimos 50 anos e poderá alcançar em 2025 o sexto lugar no ranking dos países com maior número de idosos em sua população. A hidroterapia é um método terapêutico que utiliza os princípios físicos da água em conjunto com a cinesioterapia para prevenir, manter, retardar, melhorar ou tratar as disfunçôes físicas características do envelhecimento. Objetivo: Avaliar a força muscular, equilíbrio e qualidade de vida entre idosos praticantes de hidroterapia e idosos sedentários do município de Cuiabá (MT). Materiais e métodos: Foram incluídos no estudo idosos com idade entre 60 e 90 anos, praticantes e não praticantes de hidroterapia. Foi avaliada a força muscular pela Escala de Força de Kendall, o equilíbrio por meio da Escala de Equilíbrio Funcional de Berg e a qualidade de vida pelo questionário SF-36. Foi utilizado o teste T-Student com nível de 5\% de significância para comparação dos valores de força muscular e equilíbrio entre os grupos, e para a análise da qualidade de vida foram realizadas medidas de tendência central e dispersão. Resultados: Os 19 idosos praticantes de hidroterapia obtiveram melhor desempenho quanto à força muscular, equilíbrio e qualidade de vida em comparação aos 19 idosos sedentários, sendo todas as variantes estatisticamente relevantes com p-valor $<0,05$. Conclusáo: A hidroterapia contribui de forma positiva com a melhora da qualidade de vida, equilíbrio e força muscular dos idosos praticantes.

Palavras-chave: Qualidade de vida; força muscular; equilíbrio; idosos.

\section{ABSTRACT}

Introduction: Brazil has a population of 15 million people aged 60 years or over. This population has shown a growth of $5 \%$ in the last 50 years and will reach in 2025 on the sixth place in the ranking of countries with the highest number of elderly people among its population. Hydrotherapy is a therapeutic method that use the physical principles of the water together the kinesiotherapy to prevent, keep, retard, improve or treat physical dysfunctions of the aging. Objective: Evaluate muscle strength, balance and quality of life among elderly practitioners of hydrotherapy and sedentary elderly in Cuiabá (MT). Materials and methods: Were included elderly between 60 to 90 years, practitioners and non-hydrotherapy practitioners. The muscle strength was assessed by Kendall Force Scale, balance by Functional Balance Scale of Berg and the quality of life through the SF-36 questionnaire. Was used the Student t-test with a $5 \%$ level of significance for comparison of the values between the groups, and to analyze the quality of life was used measures of central tendency and dispersion. Results: The 19 elderly practitioners of 
hydrotherapy had better performance in the muscle strength, balance and quality of life when compared the sedentary elderly group. All variables presented statistical relevance with p-value $<0.05$. Conclusion: Hydrotherapy contributes positively to improving the quality of life, balance and muscle strength in the elderly.

Keywords: Quality of life; muscle strength; balance; elderly.

\section{Introdução}

O Brasil apresenta uma população de 15 milhôes de habitantes com idade igual ou superior a 60 anos, que tem apresentado crescimento de $5 \%$ nos últimos 50 anos, e nessa tendência de crescimento poderá alcançar em 2025 o sexto lugar no ranking dos países com o maior número de idosos em sua populaçáo ${ }^{1,2}$.

Devido ao grande aumento dessa população, ressalta-se a relevância de assegurar ao indivíduo da terceira idade não somente longevidade, mas também uma boa qualidade de vida, definida pela Organização Mundial de Saúde (OMS) como "a percepção do indivíduo sobre a sua posição na vida, no contexto da cultura e dos sistemas de valores nos quais ele vive, e em relaçáa a seus objetivos, expectativas, padróes e preocupaçóes"3.

Seguindo o mesmo raciocínio da OMS, Vecchia et al. ${ }^{4}$ obtiveram em um estudo com idosos como fatores indispensáveis para o alcance da qualidade de vida a boa convivência e participação social no contexto familiar ou organizacional, adoção de hábitos saudáveis quanto à alimentação, bom sono e prática de exercícios físicos.

Dessa forma, pode-se considerar o envelhecimento biológico como um obstáculo para atingir a qualidade de vida em virtude das modificaçóes que e causa, sendo as mais evidentes observadas no sistema musculoesquelético, como perda de massa muscular, perda de densidade óssea, desgaste e desidratação das articulaçôes, diminuição da velocidade de contração muscular, declínio da propriocepção e diminuição da excitabilidade neural ${ }^{2}$.

Observadas as alteraçóes ocorridas no envelhecimento, diversos recursos sáo propostos na tentativa de amenizá-las de modo a garantir à independência funcional do idoso. Nesse contexto, a hidroterapia é um recurso fisioterapêutico que proporciona melhoras osteomioarticulares utilizando como princípios os elementos físicos da água - empuxo, viscosidade, pressão hidrostática, tensáo superficial e temperatura. $\mathrm{O}$ recurso tem como resultados a facilitação na amplitude de movimento, alívio da dor por relaxamento muscular (inibição dos receptores nociceptivos), dinamizaçáo do retorno venoso e melhora do equilíbrio devido à busca do centro de gravidade 5 .

Sendo assim, esta pesquisa teve como objetivo avaliar a força muscular, equilíbrio e qualidade de vida entre idosos praticantes de hidroterapia e idosos sedentários do município de Cuiabá (MT).

\section{Método}

Foi realizado um estudo observacional de corte transversal nos meses de setembro e outubro de 2011, comparando grupos de idosos praticantes e não praticantes de hidroterapia. Foram incluídos no estudo idosos na faixa etária de 60 a 90 anos de ambos os sexos, saudáveis e sedentários ou praticantes de hidroterapia. Foram excluídos do estudo idosos com patologias que prejudicam a mobilidade física e com alteraçôes cognitivas. Todos foram previamente informados sobre o estudo pelos pesquisadores e assinaram um termo de consentimento livre e esclarecido.

Os indivíduos foram divididos em dois grupos, sendo um grupo formado por idosos praticantes de hidroterapia no mínimo duas vezes por semana e o outro de idosos sedentários. A amostra de praticantes de hidroterapia foi obtida no Centro de Convivência Padre Firmo da cidade de Cuiabá (MT), no qual os participantes predominantemente realizavam a atividade aquática $\mathrm{e}$ esporadicamente participavam de outras atividades recreativas ofertadas no Centro, tais como bordado, pintura e dança. Já os sedentários foram selecionados por entrevista nos bairros Praeiro e Cidade Alta, localidades próximas ao Centro de Convivência.

Para a avaliação da qualidade de vida foi utilizado o questionário SF-36 (The Medical Outcomes Study 36Item Short Form Health Survey), composto de 36 itens distribuídos em oito domínios: Capacidade Funcional (CF), com 10 itens; Limitação por Aspecto Físico (LAF), com 4 itens; Dor, com 2 itens; Estado Geral de Saúde (EGS), com 5 itens; Vitalidade, com 4 itens; Aspectos Sociais, com 2 itens; Limitação por Aspecto Emocional (LAE), com 3 itens; e Saúde Mental, com 5 itens. A avaliação dos dados do questionário ocorreu primeiramente pela correspondência de cada item a seu respectivo domínio, e posteriormente cada domínio foi convertido em pontuaçóes de 0 a 100 , sendo 0 o pior valor e 100 um bom estado de saúde. A escolha desse questionário ocorreu por ser índice de percepção do status geral de saúde que incorpora padrōes comportamentais ${ }^{6,7,8}$.

Para a avaliação do equilíbrio funcional foi selecionada a Escala de Equilíbrio de Berg, pois é de fácil aplicação, baixo custo e garante a segurança física do idoso. É composta por 14 atividades funcionais com cinco itens cada e pontuação de 0-4 para cada tarefa, sendo 
que 0 corresponde a "incapaz de realizar a tarefa" e 4 a "realiza a tarefa independente", e as atividades incluíam "alcançar", "girar", "transferir-se", "permanecer em pé" e "levantar-se". O escore máximo a ser alcançado são 56 pontos, sendo referenciada a pontuaçáo menor com maior risco de quedas, estabelecendo uma relação inversamente proporcional ${ }^{9,10}$.

A Escala Muscular de Kendall foi utilizada como método de escolha para a avaliação da força dos principais grupos musculares, que varia de 0 a 5 , sendo que: $0=$ nula (ausência de contração); 1=traço (esboço de contração sem movimento); 2 =fraco (há movimento com anulação da gravidade); 3 =regular (contração e movimento contra gravidade); 4=bom (contração e movimento contra leve resistência externa); $5=$ normal (contração e movimento contra forte resistência externa) ${ }^{11}$. Os músculos avaliados no estudo foram bíceps braquial, tríceps braquial, deltóide fibras anteriores, quadríceps, isquiotibiais e tibial anterior, sendo estabelecido apenas o lado direito como parâmetro, por ser o lado dominante na amostra e apresentar maior força e habilidade devido à sua maior participação nas atividades de vida diária ${ }^{12}$.

A análise estatística foi realizada a partir do teste $\mathrm{t}$ de Student, com nível de significância de 5\% (p<0,05) para comparação da força muscular e equilíbrio entre os grupos. A análise da qualidade de vida foi realizada pelo Software Microsoft Excel 2007, obtendo-se a média, mediana, mínima, máxima e desvio padrão.

Este trabalho foi aprovado pelo Comitê de Ética em Pesquisa da Universidade de Cuiabá sob o protocolo $n^{\circ}$ 2010-228.

\section{Resultados}

Fizeram parte do estudo 38 idosos com média de $71,3 \pm 7,7$ anos de ambos os sexos, dos quais 19 eram praticantes de hidroterapia, com média de idade de $71,5 \pm 6,5$ anos, e 19 eram sedentários, com média de idade de $71 \pm 8,9$ anos.
Em relação ao sexo, 76,3\% eram mulheres, das quais $34,2 \%$ eram ativas e $42,1 \%$ sedentárias. A maioria dos idosos tinha idade menor que 75 anos (63,2\%), 57,9\% eram hipertensos e apenas 34,2\% eram diabéticos (Tabela 1).

Tabela 1: Perfil dos idosos ativos e sedentários por sexo, idade, HAS e diabetes mellitus. Cuiabá (MT), 2011.

\begin{tabular}{lccc}
\hline \multirow{2}{*}{ Características } & \multicolumn{2}{c}{ Idosos } & \multirow{2}{*}{ Total } \\
\cline { 2 - 3 } & Ativos & Sedentários & \\
\hline Sexo & & & \\
Masculino & $15,8 \%$ & $7,9 \%$ & $23,7 \%$ \\
Feminino & $34,2 \%$ & $42,1 \%$ & $76,3 \%$ \\
Idade & & & \\
$\leq 75$ anos & $34,2 \%$ & $28,9 \%$ & $63,2 \%$ \\
$>75$ anos & $15,8 \%$ & $21,1 \%$ & $36,8 \%$ \\
HAS & & & \\
Sim & $26,3 \%$ & $31,6 \%$ & $57,9 \%$ \\
Não & $23,7 \%$ & $18,4 \%$ & $42,1 \%$ \\
Diabético & & & \\
Sim & $15,8 \%$ & $18,4 \%$ & $34,2 \%$ \\
Não & $34,2 \%$ & $31,6 \%$ & $65,8 \%$ \\
Total & $50,0 \%$ & $50,0 \%$ & $100,0 \%$ \\
\hline
\end{tabular}

Legenda: HAS - Hipertensão arterial sistêmica.

Em relação à qualidade de vida, os idosos ativos obtiveram pontuaçôes maiores em sete dos oito domínios avaliados quando comparados aos idosos sedentários. $\mathrm{O}$ domínio Capacidade Funcional (CF) apresentou média de 73,16 $\pm 23,11$ no Grupo Ativo (GA) e de 59,21 $\pm 26,68$ no Grupo Sedentário (GS), assim como as Limitaçóes por Aspecto Físico (LAF) apresentaram média de $72,37 \pm 35,25$ no GA e 32,89 $\pm 40,01$ no GS. A dimensão de Dor apresentou média de 69,68 $\pm 30,25$ pontos nos idosos praticantes de hidroterapia e de 44,21 $\pm 32,64$ nos não praticantes (Tabelas 2 e 3). O domínio de Estado Geral de Saúde (EGS) não foi estatisticamente diferente entre os grupos GS e GA (Tabelas 2 e 3).

Em relação ao equilíbrio dos idosos, os indivíduos que praticam a hidroterapia apresentaram um melhor

Tabela 2: Distribuição dos dados de idosos ativos segundo as categorias do SF-36. Cuiabá (MT), 2011.

\begin{tabular}{lcccccccc}
\hline Estatísticas & CF* & LAF* & DOR & EGS* & Vitalidade & $\begin{array}{c}\text { Aspecto } \\
\text { social }\end{array}$ & LAE* & Saúde Mental \\
\hline Média & 73,16 & 72,37 & 69,68 & 57,16 & 76,58 & 88,82 & 71,93 & 80,21 \\
Mediana & 85,00 & 100,00 & 72,00 & 57,00 & 85,00 & 100,00 & 100,00 & 80,00 \\
Moda & 85,00 & 100,00 & 100,00 & 52,00 & 90,00 & 100,00 & 100,00 & 96,00 \\
Desvio Padrão & 23,11 & 35,25 & 30,25 & 14,60 & 21,22 & 23,53 & 40,47 & 15,35 \\
Coeficiente de & $31,6 \%$ & $48,7 \%$ & $43,4 \%$ & $25,5 \%$ & $27,7 \%$ & $26,5 \%$ & $56,3 \%$ & $19,1 \%$ \\
variação & 70,00 & 100,00 & 80,00 & 55,00 & 80,00 & 75,00 & 100,00 & 52,00 \\
Intervalo & 100,00 & 100,00 & 100,00 & 80,00 & 100,00 & 100,00 & 100,00 & 100,00 \\
Máximo & 30,00 & 0,00 & 20,00 & 25,00 & 20,00 & 25,00 & 0,00 & 48,00 \\
Mínimo & & & & & & & & \\
\hline
\end{tabular}

CF - Capacidade Funcional; EGS - Estado Geral de Saúde; LAF - Limitação por Aspecto Funcional; LAE - Limitaçáo por Aspecto Emocional. 
Tabela 3: Distribuição dos dados de idosos sedentários segundo as categorias do SF-36. Cuiabá (MT), 2011.

\begin{tabular}{lcccccccc}
\hline Estatísticas & CF & LAF & DOR & EGS & Vitalidade & Aspecto social & LAE & $\begin{array}{c}\text { Saúde } \\
\text { Mental }\end{array}$ \\
\hline Média & 59,21 & 32,89 & 44,21 & 60,42 & 60,53 & 72,11 & 52,63 & 66,11 \\
Mediana & 65,00 & 25,00 & 40,00 & 62,00 & 55,00 & 87,50 & 33,33 & 60,00 \\
Moda & 65,00 & 0,00 & 10,00 & 72,00 & 55,00 & 87,50 & 100,0 & 60,00 \\
Desvio Padráo & 26,68 & 40,01 & 32,64 & 13,67 & 29,29 & 25,20 & 42,04 & 21,64 \\
Coeficiente de & $45,1 \%$ & $121,6 \%$ & $73,8 \%$ & $22,6 \%$ & $48,4 \%$ & $34,9 \%$ & $79,9 \%$ & $32,7 \%$ \\
variaçáo & 95,00 & 100,00 & 100,00 & 47,00 & 95,00 & 75,00 & 100,00 & 76,00 \\
Intervalo & 95,00 & 100,00 & 100,00 & 82,00 & 100,00 & 100,00 & 100,00 & 100,00 \\
Máximo & 0,00 & 0,00 & 0,00 & 35,00 & 5,00 & 25,00 & 0,00 & 24,00 \\
Mínimo & & & & & & & &
\end{tabular}

CF - Capacidade Funcional; EGS - Estado Geral de Saúde; LAF - Limitação por Aspecto Funcional; LAE - Limitação por Aspecto Emocional.

equilíbrio estático e dinâmico quando comparado ao grupo de sedentários, com uma média de pontuação de $49,6 \pm 5,04$ versus média de $43,4 \pm 8,97$ dos sedentários, tendo como categorias: Base de Sustentação, Componentes Rotacionais, Alcance Funcional, Provas Estacionárias e Transferências, as quais apresentaram diferença das médias entre elas de 6,2, sendo esses resultados estatisticamente significativos com $\mathrm{p}<0,05$ (Tabela 4).
Quanto à força muscular, os idosos ativos obtiveram valores acima da graduação 4 de força muscular em todos os grupos musculares avaliados, e o grupo de sedentários, em contrapartida, valores inferiores a $4 \mathrm{em}$ todas as categorias, com significância estatística para ambos os grupos $(p<0,05)$, podendo entáo afirmar com $95 \%$ de confiança que as médias entre os grupos de idosos ativos e sedentários são diferentes (Tabela 5).

Tabela 4: Comparação das médias de pontuação na Escala de Berg entre as categorias, segundo grupos de tarefas da Escala Berg. Cuiabá (MT), 2011.

\begin{tabular}{|c|c|c|c|c|c|}
\hline \multirow{2}{*}{$\begin{array}{l}\text { Grupos de tarefa Escala } \\
\text { de Berg }\end{array}$} & \multicolumn{2}{|c|}{ Média das pontuaçóes em cada categoria } & \multirow{2}{*}{$\begin{array}{l}\text { Pontuaçáo } \\
\text { máxima }\end{array}$} & \multirow{2}{*}{$\begin{array}{c}\text { Diferença das } \\
\text { médias entre as } \\
\text { categorias }\end{array}$} & \multirow{2}{*}{$\begin{array}{l}\text { Perda entre as } \\
\text { categorias em } \\
\text { relação à pontua- } \\
\text { çâo maxima }\end{array}$} \\
\hline & Ativos & Sedentários & & & \\
\hline Todos os grupos & 49,6 & 43,4 & 56 & 6,2 & $11,1 \%$ \\
\hline Base de sustentaçáo & 8,6 & 6 & 12 & 2,6 & $22,4 \%$ \\
\hline $\begin{array}{l}\text { Componentes } \\
\text { rotacionais }\end{array}$ & 10,9 & 9,8 & 12 & 1,0 & $8,8 \%$ \\
\hline Alcance funcional & 3,3 & 3 & 4 & 0,3 & $7,9 \%$ \\
\hline Provas estacionárias & 15,5 & 14,2 & 16 & 1,3 & $8,2 \%$ \\
\hline Transferências & 10,9 & 10,2 & 12 & 0,6 & $5,7 \%$ \\
\hline
\end{tabular}

Tabela 5: Comparação das médias das pontuações entre os idosos ativos e sedentários segundo os músculos do lado direito. Cuiabá (MT), 2011

\begin{tabular}{lccc}
\hline \multirow{2}{*}{ Músculos } & \multicolumn{2}{c}{ Média das pontuaçóes } & P - valor \\
\cline { 2 - 3 } & Ativos & Sedentários & 0,0008 \\
Isquiotibiais & $4,3 \pm 0,6$ & $3,4 \pm 0,9$ & 0,0012 \\
Quadríceps & $4,7 \pm 0,5$ & $3,8 \pm 1,0$ & 0,0061 \\
Tibial anterior & $4,5 \pm 0,6$ & $3,8 \pm 0,9$ & 0,0135 \\
Bíceps braquial & $4,6 \pm 0,6$ & $3,8 \pm 1,2$ & 0,0162 \\
Tríceps braquial & $4,5 \pm 0,6$ & $3,7 \pm 1,2$ & 0,0396 \\
Deltóide anterior & $4,1 \pm 0,8$ & $3,4 \pm 1,2$ & \\
\hline
\end{tabular}




\section{Discussão}

As variáveis relacionadas neste estudo demonstraram claramente a relevância do exercício físico para alavancar a qualidade de vida, ainda que na faixa etária abordada as características fisiológicas sejam desfavoráveis para a sua prática. As características da população divergiram em cada regiáo, mas em um aspecto geral o grupo representou cerca de $7,4 \%$ da populaçáo do Brasil, sendo que na região Centro-Oeste correspondeu a 5,8\%, segundo censo $2010^{13}$.

Em relação ao sexo, observou-se uma maior prevalência de mulheres, como na amostra de pesquisa de Figliolino et al., ${ }^{11}$ em que analisaram a influência do exercício físico em idosos relacionando-o com o equilíbrio, marcha e atividade de vida diária. Foi observada uma melhora relevante do ponto de vista estatístico na Escala de Tinetti, com $\mathrm{p}<0,05$ em relação à marcha e ao equilíbrio, em que idosos praticantes de atividade física superaram a pontuação. Além disso, na escala de Katz todos os idosos foram classificados como independentes e, embora na Atividade de Vida Diária (AVD) o grupo praticante tenha obtido pontuação maior, não foi estatisticamente significante.

Corroborando com esta pesquisa, Januário et al. ${ }^{14}$, ao analisarem a qualidade de vida de idosos sedentários e ativos, verificaram que $80 \%$ de sua amostra de 102 idosos era do gênero feminino, e após a divisão da amostra em Grupo de Atividade Física (GAT) e Grupo Controle (GC) perceberam diferenças estatísticas importantes e resultados inferiores em quase todos os domínios do SF-36 quanto ao GC, exceto quanto aos parâmetros de limitação física e emocional.

Nesta pesquisa, apesar da prevalência maior de mulheres quando comparado o grupo de idosos ativos e sedentários, verificou-se que a maioria dos homens avaliados eram ativos, o que pode ser explicado pela maioria dos homens trabalharem até mais avançada idade que as mulheres.

Quando avaliada a presença de comorbidades associadas ao envelhecimento, constata-se que quase todos os idosos participantes relataram a presença de diabetes mellitus, hipertensão arterial sistêmica (HAS) ou ambos. Esse resultado acompanha os dados obtidos por Mendonça et al. ${ }^{15}$, que analisando o perfil de uma amostra de 44 idosos frequentadores de um parque em São Paulo quanto ao risco cardiovascular, aptidáo física e prática de atividade física concluíram que $91 \%$ praticava atividade física, e quanto aos fatores de riscos $70 \%$ tinham pelo menos um fator (2\% HAS, $14 \%$ diabetes mellitus, $23 \%$ hipercolesterolemia, $16 \%$ obesidade e $9 \%$ sedentarismo).

No estudo realizado por Pimenta et al. ${ }^{16}$, quando avaliada a qualidade de vida entre aposentados, verificou-se que entre as patologias mais relatadas estavam a HAS (39\%), depressão $(24,1 \%)$, seguida por diabetes mellitus $(10 \%)$ e doenças articulares (9\%).

Sabe-se que o idoso possui fatores intrínsecos (fisiológicos, emocionais etc.) que levam ao declínio do seu bem estar subjetivo ${ }^{9,14}$, e, partindo-se dessa premissa, este estudo buscou a prática de hidroterapia como fator potencialmente benéfico para a melhora do equilíbrio, força muscular e, consequentemente, da qualidade de vida da população estudada.

Quando analisados os resultados do SF-36 nesta pesquisa, os idosos ativos obtiveram pontuações maiores em sete dos oito domínios avaliados quando comparados aos idosos sedentários, caracterizando melhor qualidade de vida para o grupo de idosos ativos. O domínio Capacidade Funcional (CF) foi maior no Grupo Ativo (GA) $(73,16 \pm 23,11)$ do que no Grupo Sedentário (GS) $(59,21 \pm 26,68)$, assim como as Limitaçóes por Aspectos Físicos (LAF) também foram superiores no GA $(72,37 \pm 35,25)$ do que no GS $(32,89 \pm 40,01)$. Além disso, a moda nos ativos foi de 100 enquanto o escore dos sedentários foi de 25 .

Quanto à dimensão Dor foram encontrados resultados semelhantes aos descritos acima, caracterizando que os idosos praticantes de hidroterapia apresentaram menor dor $(69,68 \pm 30,25)$ em relação aos idosos sedentários $(44,21 \pm 32,64)$, e a moda apresentando valores ainda mais discrepantes entre os grupos, sendo de 100 no GA e de 10 no GS. O efeito analgésico que a hidroterapia em água aquecida promove é devido à estimulação sensorial elevada pela pressão hidrostática, turbulência e temperatura da água, além de modular as fibras sensitivas relativas a tato, pressão e temperatura, mascarando a dor. Outro fator que pode ter contribuído para a analgesia em meio aquático foi o convívio social que atua como fator para distração do quadro álgico, justificando a menor prevalência de dor no grupo ativo ${ }^{17,18,19}$.

No estudo realizado por Mota et al. ${ }^{7}$, ao compararem idosos que praticavam exercício físico regularmente e que não praticavam, concluíram que o grupo ativo teve um desempenho superior em todos os domínios do questionário SF-36 em relação ao grupo controle. Seguindo a mesma linha de pensamento, Toscano e Oliveira $^{20}$, em seu estudo de associação do nível de atividade física à qualidade de vida de idosas, chegaram à conclusão de que as mais ativas superaram todas as dimensôes do SF-36 quando comparadas às idosas com menor nível de atividade física.

$\mathrm{O}$ único domínio em que não se observou diferença relevante entre GS e GA foi Estado Geral de Saúde (EGS). Pimenta et al. ${ }^{16}$, em sua análise de grupos de aposentados, referiram que portadores de diabete mellitus apresentaram tendência relevante de menor escore para o domínio EGS. Castro et al. ${ }^{21}$ definem Estado 
Geral de Saúde como uma percepção subjetiva da saúde global, e em sua pesquisa referente à qualidade de vida dos pacientes com insuficiência renal constataram que os pacientes com diabete mellitus demonstram menor pontuação para dimensão EGS, acrescentando que a presença de doenças crônicas, a idade avançada e tratamento contínuo e as possíveis complicações podem alterar a visão que os indivíduos possuem de seu EGS. Tal fato pode justificar o resultado obtido no presente estudo que conta com um perfil similar quanto à presença de comorbidades.

Quando se avaliou o equilíbrio dos idosos utilizando a Escala de Berg, verificou-se que os indivíduos praticantes de hidroterapia apresentaram melhor equilíbrio estático e dinâmico quando comparados com o grupo de sedentários. Tais resultados podem ser justificados pelas propriedades positivas do meio aquático, que permite o atraso da queda e exige reaçóes de equilíbrio para a manutenção da simetria corporal, ativando o sistema musculoesquelético (especialmente os músculos antigravitacionais), sensorial e nervoso. Além disso, observa-se que a repetiçáo dos movimentos favorece a plasticidade neural, garantindo o aperfeiçoamento do equilíbrio e desenvoltura das cadeias musculares ${ }^{22}$.

Corroborando esta pesquisa, Bruni et al. ${ }^{23}$ avaliaram o equilíbrio e marcha de 24 idosos, sendo 11 do grupo que realizou 10 sessóes de hidroterapia uma vez por semana e 13 do grupo controle, que recebeu orientaçóes sobre prevenção de quedas por meio de duas palestras e uma cartilha sobre o assunto e obteve melhora significativa do equilíbrio dos idosos participantes da hidroterapia, o que não foi observado no grupo controle.

Sabendo-se que o equilíbrio musculoesquelético sofre influência dos sistemas visual, vestibular e somato-sensorial, observou-se que esses três precursores estão afetados durante o envelhecimento por um processo natural de degradação dos sistemas, aumentando a instabilidade do idoso ${ }^{24}$. O treino do equilíbrio é atenuado pela propriedade de refraçáo do meio líquido, havendo distorção na visualização dos movimentos realizados, que exige menor atençáo visual durante a execuçáo dos exercícios, compensando a falha do sistema visual ${ }^{18}$.

Isso também foi observado no estudo de Resende et al. ${ }^{9}$, que avaliaram o equilíbrio de idosas inseridas no programa de hidroterapia e verificaram no intervalo de 12 semanas a melhora nos resultados da Escala de Berg. O estudo confirma a eficácia da atividade aquática, visto que o melhor resultado na Escala de Berg traz a redução do risco de quedas, as quais predispóem os idosos a fraturas e lesóes musculoesqueléticas, acarretando imobilismo, altos custos com o tratamento e o medo de cair novamente, sendo um obstáculo para sua qualidade de vida.

Entre as alteraçóes fisiológicas do envelhecimento, as relacionadas ao sistema musculoesquelético (perda gradual da massa muscular, força, potência e flexibilidade) são as que acarretam maior limitação funcional e levam ao ciclo quedas-dor-medo-imobilidade ${ }^{11}$.

No trabalho de Candeloro e Caromano 5 foi realizado um estudo com 15 idosas participantes de um programa de hidroterapia e outras 16 idosas do grupo controle, das quais foram avaliadas a força muscular por meio da miometria e a flexibilidade pelos testes de envergadura e flexão anterior do tronco. Observou-se melhora da flexibilidade e força muscular das idosas após conduta na hidroterapia, ressaltando que o treinamento de força ideal para os idosos deve ser de baixa a média intensidade e baixo impacto e longa duração, condições encontradas na conduta hidroterápica. Para os resultados favoráveis ao aumento da força muscular, as propriedades físicas da água de densidade relativa, flutuação e viscosidade, segundo estudos, são os facilitadores desses valores, visto que reduzem a sobrecarga articular e favorecem à atuação equilibrada dos músculos, facilitando a realização de exercícios com qualidade em maiores amplitudes do que seriam possíveis no solo ${ }^{25}$.

Em relação ao escore baixo do grupo sedentário quanto à força muscular, a literatura justifica que tais declínios ocorrem pelo desuso, que somado ao processo de envelhecimento acelera as reduçóes de massa muscular, potência e agilidade corporal ${ }^{26}$.

Sendo assim, o aumento da força muscular através da hidroterapia torna-se um fator adicional que justifica a melhora do equilíbrio e qualidade de vida também verificada neste estudo, uma vez que o treinamento muscular melhora a habilidade de gerar força muscular, especialmente em membros inferiores, precipitando a melhora do equilíbrio e, consequentemente, da independência funcional do indivíduo ${ }^{27}$.

Além disso, compreendendo-se as alteraçôes fisiológicas do envelhecimento e o empecilho que representam para a autonomia social, observou-se que o treino da força muscular melhora a habilidade e agilidade dos movimentos, cuja qualidade facilita a marcha, manutenção do equilíbrio e execução de atividades da vida diária, prevenindo limitaçóes funcionais e evoluçóes para quedas, cujas consequências podem levar ao imobilismo corporal, trazendo sofrimento e altos custos com tratamento de saúde ${ }^{24}$.

Entretanto, a amostra estudada de anciáos ativos praticava esporadicamente atividades recreativas, incluindo dança, que induz a excitação física e mental e o bom humor, fatores que também podem ter favorecido à melhor pontuação desse grupo em quase todas as variáveis avaliadas neste estudo ${ }^{28}$.

Ainda acerca da amostra pesquisada, houve prevalência significativa de idosos com patologias crônicas associadas, o que levanta novamente o debate para a questão da senilidade e novas portas para a realização 
de pesquisas para afirmar se a hidroterapia pode atuar sozinha não apenas como recurso facilitador, mas como fator preventivo.

\section{Conclusão}

Este estudo demonstrou que a qualidade de vida, equilíbrio e força muscular dos idosos praticantes de hidroterapia são melhores do que os sedentários. Mesmo sabendo que os resultados desta pesquisa apontam para a adoção da hidroterapia como fator positivo na busca do envelhecimento com qualidade, sugerem-se novos estudos em virtude da pequena amostra avaliada e do fato de os idosos praticarem esporadicamente outras atividades recreativas oferecidas pelo centro de convivência.

\section{Agradecimentos}

Agradecemos ao Centro de Convivência Padre Firmo da cidade de Cuiabá (MT) e a todos os idosos participantes desta pesquisa.

\section{Referências}

1. Trentini CM. Qualidade de vida em idosos. [tese de doutorado]. Porto Alegre: Faculdade de Medicina, Universidade Federal do Rio Grande do Sul. Programa de pós-graduaçáo em ciências médicas: psiquiatria; 2004.

2. Marchi Netto FL. Aspectos biológicos e fisiológicos do envelhecimento humano e suas implicaçóes na saúde do idoso. Pensar a Pratica. 2004;7:75-84.

3. Seidl EMF, Zannon CMLC. Qualidade de vida e saúde: aspectos conceituais e metodológicos. Cad Saúde Pública. 2004;20(2):580-588.

4. Vecchia RD, Ruiz T, Bocchi SM, Corrente JE. Qualidade de vida da terceira idade: um conceito subjetivo. Rev Bras Epidemiologia. 2005;8(3):246-252.

5. Candeloro JM, Caromano FA. Efeito de um programa de hidroterapia na flexibilidade e na força muscular de idosas. Rev Bras Fisioterapia. 2007;11(4):303-309.

6. Carvalho Filho ET, Netto MP, Passini U. In: Filho ETC. Geriatria Fundamentos, clínica e terapêutica. 2a ed. São Paulo: Atheneu. 2005. 193-210.

7. Mota J, Ribeiro JL, Carvalho J, Matos MG. Atividade física e qualidade de vida associada à saúde em idosos participantes e não participantes em programas regulares de atividade física. Rev bras Educ Fís Esp. 2006;20(3):219-225.

8. Coelho ESP, Pedroso MFBP. Avaliação da qualidade de vida em idosos residentes no município de Santos / SP. Rev Ceciliana. 2012;4(1):4-8.
9. Resende SM, Rassi CM, Viana FP. Efeitos da hidroterapia na recuperação do equilíbrio e prevenção de quedas em idosas. Rev Bras Fisioterapia. 2008;12(1):57-63.

10. Pereira VV, Maia RA, Silva SMCA. The functional assessment Berg Balance Scale is better capable of estimating fall risk in the elderly than the posturographic Balance Stability System. Arq Neuro-Psiquiatr. 2013;71(1):5-10.

11. Figliolino JAM, Morais TB, Berbel AM, Corso SD. Análise da influência do exercício físico em idosos com relação a equilíbrio, marcha e atividade de vida diária. Rev Bras Geriatria e Gerontologia. 2009;12(2):227-238.

12. Carvalho J, Oliveira J, Magalhães J, Ascensão A, Mota J, Soares JMC. Força muscular em idosos II - Efeito de um programa complementar de treino na força muscular de idosos de ambos os sexos. Rev Portuguesa de Ciências do Desporto. 2004;4(1):58-65.

13. Fernandes APS, Oliveira RC. O idoso e a ludicidade. Publ. UEPG Humanit. Sci Linguist Lett Arts 20(2):151-160, jul./dez. 2012. Disponível em: <http://www.revistas2.uepg. br/index.php/humanas>. [acesso em: 20 mar. 2013].

14. Januário RSB, Junior HS, Liutti MC, Decker D, Molari M. Qualidade de vida em idosos ativos e sedentários. ConScientiae Saúde 2011;10(1):112-121.

15. Mendonça TT, et al. Risco cardiovascular, aptidáo física e prática de atividade física de idosos de um parque de São Paulo. Rev Bras Ci e Mov. 2004;12(2):19-24.

16. Pimenta F, Simil FF, Tôrres HOG, Amaral CFS, Rezende $\mathrm{CF}$, et al. Avaliação da qualidade de vida de aposentados com a utilização do questionário SF-36. Rev Assoc Med Bras. 2008;54(1):55-60.

17. Caromano FA, Candeloro JM. Fundamentos da Hidroterapia para idosos. Arq Ciênc. Saúde Unipar. 2001;5(2):187-195.

18. Becker BE, Ruoti RG, Morris DM, Cole A. Reabilitação aquática. São Paulo: Manole; 2000.

19. Norm A, Hanson B. Exercícios aquáticos terapêuticos. $1^{\mathrm{a}}$ ed. São Paulo: Manole;1998.

20. Toscano JJO, Oliveira ACC. Qualidade de Vida em Idosos com Distintos Níveis de Atividade Física. Rev Bras Med Esporte. 2009;15(3):169-173.

21. Castro M, Caiuby AVS, Draibe AS, Canziani MEF. Qualidade de vida de pacientes com insuficiência renal crônica em hemodiálise avaliada através do instrumento genérico SF-36. Rev Assoc Med Bras. 2003;49(3):245-249.

22. Gabilan YPL, Perracini MR, Munhoz MSL, Ganança FF. Fisioterapia Aquática para Reabilitação Vestibular. Rev Acta Orl. 2006; 24(1): 25-30.

23. Bruni BM, Granado FB, Prado RA. Avaliação do equilíbrio postural em idosos praticantes de hidroterapia em grupo. Rev O Mundo da Saúde. 2008;32(1):56-63.

24. Ruwer LS, Rossi AG, Simon LF. Equilíbrio no idoso. Rev Bras Otorrinolaringologia. 2005;71(3):298-303. 
25. Carregaro RL, Toledo AM. Efeitos fisiológicos e evidências científicas da eficácia da fisioterapia aquática. Rev Mov. 2008;1(1):23-27.

26. Teixeira D, Junior S, Lima D, Gomes S, Brunetto A. Efeitos de um programa de exercício físico para idosas sobre variáveis neuro-motoras, antropométrica e medo de cair. Rev Bras Fís Esp. 2007;21(2):107-120.
27. Douris P, Southard V, Varga C, Schauss W, Gennaro C, Reiss A. The Effect of Land and Aquatic Exercise on Balance Scores in Older Adults. Journal of Geriatric Physical Therapy. 2003;26(1):3-6.

28. Oliveira LC, Pivoto EA, Vianna PCP. Análise dos resultados de qualidade de vida em idosos praticantes de dança sênior através do SF-36. Rev Acta Fisiatr. 2009;16(3):101-104.

Como citar este artigo:

Salicio VAM, Mattos TS, Brandalise VLA, Bittencourt WS, Salicio MA. Estudo comparativo da força muscular, equilíbrio e qualidade de vida entre idosos praticantes de hidroterapia e idosos sedentários do município de Cuiabá (MT). Rev. Aten. Saúde. 2015;13(46):23-30 